\title{
Breast Cancer is not Just One Disease: A special focus of molecular heterogeneity of Triple Negative Breast Cancer
}

\author{
Sahar Mostafa GabAllah and Rana Ahmed Youness* \\ Pharmaceutical Biology Department, Faculty of Pharmacy and Biotechnology, German University in Cairo, Cairo, Egypt \\ *Corresponding author: Rana Ahmed Youness, Pharmaceutical Biology Department, Faculty of Pharmacy and \\ Biotechnology, German University in Cairo, Cairo, Egypt
}

\section{ARTICLE INFO}

Received: 幽 July 18, 2020

Published: 幽 July 27, 2020

Citation: Sahar Mostafa GabAllah, Rana Ahmed Youness. Breast Cancer is not Just One Disease: A special focus of molecular heterogeneity of Triple Negative Breast Cancer. Biomed J Sci \& Tech Res 29(1)2020. BJSTR. MS.ID.004757.

\section{ABSTRACT}

In oncology, it is not "One Size" fits all. After decades of research, a new hypothesis is recently dominating the field of oncology which is "tumors are not alike". breast cancer (BC) is a perfect example of such heterogeneous tumors. Triple Negative Breast Cancer (TNBC) is one of the most aggressive subtypes of BC. It is complex \& heterogeneous in nature; there is no available targeted therapy, as it lacks expression of any receptors (ER, PR \& HER2) which render it resists any hormonal therapy used in treatment of other subtypes of $\mathrm{BC}$. That is why this type of cancer needs especial kind of therapy or even combinational therapies. In this review, we focus on the TNBC patients and the currently available treatment protocols for those patients. Also, a special focus on the altered molecular pathways in TNBC tumors and their naturally derived and synthetic molecular weapons has been specifically tackled in this review.

\section{Introduction}

\section{Breast Cancer}

Breast Cancer (BC) is a complex heterogeneous disease which is molecularly sub-classified into several subtypes as shown in Table 1 [1]. It is one of the most leading causes of women death worldwide especially in developing countries. It is the most common cancer worldwide and the 2nd cause of cancer-related deaths in American woman after lung cancer. According to WHO, about 2.1 million annually are newly diagnosed with BC and 167.000 deaths has been reported in 2018 [2]. This represents 15\% of all cancer deaths among women. Most BC tumors originate in the breast tissue itself. This either takes place in glands for milk production (lobules) and which is called "Lobular Carcinoma"; or in ducts which is called "Ductal Carcinoma". Identification of the molecular subtype of each patient makes a great difference; it is not only important in evaluating the disease prognosis, but also, in selecting the optimum treatment regimen. Consequently, the maximum patients' response rates and overall survivals are obtained [3].
Table 1: Molecular Subtypes of Breast Cancer Patients.

\begin{tabular}{|c|c|}
\hline Breast Cancer Subtype & Molecular Profile \\
\hline Luminal A & $\begin{array}{c}\mathrm{ER}^{+} \text {and/or PR }{ }^{+}, \mathrm{HER}-2{ }^{-} \text {, and low Ki-67 } \\
(<14 \%)\end{array}$ \\
\hline Luminal B & $\begin{array}{c}\mathrm{ER}^{+} \text {and/or PR }{ }^{+} \text {and HER-2 } 2^{+} \text {(luminal- } \\
\text { HER2 group) }\end{array}$ \\
\hline HER-2 Enriched & $\mathrm{ER}^{-}, \mathrm{PR}^{-}$, and HER-2 ${ }^{+}$ \\
\hline Triple Negative Breast Cancer & ER', PR and HER-2 \\
\hline
\end{tabular}

\section{Triple Negative Breast Cancer (TNBC)}

TNBC comprises a heterogeneous group of tumors that is considered one of the most aggressive $\mathrm{BC}$ subtypes with the poorest prognosis compared to other BC subtypes [4]. Therefore, TNBC will be the focus of our review. The absence of hormonal receptors, as well as, HER-2 receptors renders it as an unresponsive tumor to hormonal therapies or therapies targeting HER2 protein. Almost $15-25 \%$ of BC women are diagnosed with TNBC. Its relapse rates greatly differ from other $\mathrm{BC}$ subtypes as TNBC relapse rates 
are the highest in the first 3-5 years. Nonetheless, it drops sharply afterwards; it even may become below that of hormone positive subtypes (Luminal A/B). TNBC is usually classified as a "basal- type" cancer; however, many studies have been done for more deep classification of TNBC using gene expression profiling as shown in Table 2 [5-8].

Table 2: Triple Negative Breast Cancer Classification.

\begin{tabular}{|c|c|c|}
\hline References & Subtypes of TNBC & Type of Classification \\
\hline$[5]$ & $\begin{array}{c}\text { Seven distinct subtypes, six are stable : Basal like1(BL1), Basal like } 2 \text { (BL2), } \\
\text { Immunomodulatory (IM), Mesenchymal (M), Mesenchymal Stem Like (MSL) \& Luminal } \\
\text { Androgen Receptor (LAR) besides one Unstable subtype (UNS) }\end{array}$ & Lehmann study \\
\hline$[6]$ & $\begin{array}{l}\text { He applies nonnegative matrix factorization \& define Four subtypes of TNBC: Basal like } \\
\text { immune active, basal like immune suppressed, Mesenchymal \& Luminal AR }\end{array}$ & Burstein et al. study \\
\hline [7] & $\begin{array}{l}\text { Using data collected from clinical \& gene expression profiles of } 494 \text { TNBC breast tumor } \\
\text { samples, the study shows four subtypes of TNBC: Luminal-Androgen receptor (LAR), } \\
\text { Basal, Claudin-low \& Claudin-high. Further classification for Luminal subtype was done } \\
\text { (based on its immunology status) into Luminal-immune positive [with better prognosis] } \\
\text { \& Luminal-immune negative. This classification enables researchers to predict tumor } \\
\text { response to immune therapy }\end{array}$ & Guillermo Prado-Vazquez \\
\hline [8] & $\begin{array}{c}\text { Study classifies TNBC microenvironment into three clusters: } \\
\text { Immune desert cluster: low cell infiltration, cannot attract immune cells \& show } \\
\text { "MYC" amplification. Innate immune -inactivated: has inactive innate immune } \\
\text { cells \& nonimmune stromal cell filtration. It has low tumor Ag presentation (which } \\
\text { may lead to immune escape) \& has a mutations in PI3K-AKT signaling pathway. } \\
\text { Immune inflamed: rich in innate \& adaptive immune cells infiltration with high } \\
\text { level of immune check point molecules which render it the most affected cluster with } \\
\text { immunotherapy strategies. }\end{array}$ & Yi Xiao \\
\hline
\end{tabular}

\section{Conventional Therapeutic Approaches for TNBC Patients}

Chemotherapy still represents the mainstay treatment for TNBC patients as a result of lacking receptors that can be treated by targeted therapy [9]. Neo-adjuvant chemotherapy is used as the first line of treatment for TNBC patients, especially for cases where surgery is contraindicated [10]. Nonetheless, this treatment approach gives higher pathological complete response (PCR) in TNBC patients rather than in HR+ BC patients [11]. It has been found that the PCR rate in basal-like1 subtype is greater than in basal-like 2 \& LAR receptor subtypes. Furthermore, TNBC patients have lower than three year progression free survival (PFS) and overall survival (OS), according to J.M. Lebert study [12]. The best neo-adjuvant therapeutic regimen for TNBC patients has not been identified yet. However, it has been found that in addition of Carboplatin results in higher rates of (PCR). But, unfortunately, it leads to greater toxicity effects.

\section{Adjuvant Therapy}

This regimen is recommended to be used for TNBC tumor with size larger than $0.5 \mathrm{~cm}$ because of their aggressiveness. The guidelines of European Society for Medical Oncology (ESMO) recommended not using adjuvant treatment if there is a residual disease after the completion of neoadjuvant treatment. However, this indication is completely reversed after the "create-x" trial results [13]. The optimal adjuvant option for TNBC is still not identified despite the use of chemotherapy containing anthracyclines and taxanes that are supported by European society guidelines [14]. Using Platinum based chemotherapy in metastatic TNBC usually recommended rather than non-platinum based chemotherapy whose use results in longer PFS in patients with no differences in their OS rates.

\section{Molecular Signaling Pathways Underlying the Molecular Heterogeneity of TNBC and Their Potential Molecular Targeting Weapons}

Different signaling pathways contribute to TNBC development, metastasis, and resistance to current therapeutic regimens [15]. $\mathrm{NF}-\kappa \mathrm{B}$ is greatly expressed in TNBC cells [16], it is the key regulator of TNBC cells apoptosis, angiogenesis, and resistance. It is one of soy isoflavones agents "Genistein" down regulate the expression of "BCL-2, BCL-xL and Cyclin B1" proteins and lead to TNBC cells apoptosis [17]. "Plumbagin" also induces apoptosis in TNBC cells through inactivation of DNA binding activity of NF- $\kappa B$ and BCL-2 and has no effect on normal breast cancer cells [18]. "Fenofibrate" cause apoptosis to TNBC cells through up regulation of "Bad", activation of "Caspase3" and down regulation of "BCL-xL" [19].

\section{JAK/STAT signaling pathway}

STAT3 overexpressed in TNBC tissues giving higher aggressiveness and poor prognosis [20]. JAK2 gene greater expressed in TNBC cells following chemotherapy indicating that it has a role in developing chemo-resistance in TNBC cells. Therefore, using JAK2 specific inhibitor "BSK805" with chemotherapy in mice cause tumor regression [21]. Drugs inhibit STAT3 (as Metformin) control tumor growth [22].

\section{PI3K/AKT/mTOR Signaling Pathway}

PI3K is over expressed in $60 \%$ of TNBC which leads to deletion or mutation in PTEN gene. AKT regulates "Bad" and thus can controls TNBC cells apoptosis [23]. PI3K/AKT pathway activation in TNBC cells which has been knocked down from ELK3 protein leads 
to increase the chemosesitivity of cells to doxorubicin [24]. Using PI3K/AKT inhibitor as "Buparlisib" activates ERK and MEK1, which decrease expression of BRCA1. "Everolimus" (mTOR inhibitor) has antitumor activity against TNBC cancer cells [25].

\section{Wnt/ $\beta$-Catenin Signaling Pathway}

Wnt/ $\beta$-catenin is associated with poor prognosis in TNBC cells [26]. Using "ICRT-3" (Wnt/ $\beta$-catenin pathway inhibitor) has been reported to inhibit TNBC cells proliferation [27]"Salinomycin"(anticancer drug) induces degradation of RLP6 (Wnt/ $\beta$-catenin co-receptor) inhibiting TNBC cells' proliferation [28].

\section{Hedgehog signaling pathway}

The dysregulation of Hedgehog signaling pathway (Hh) enhances invasion and migration as well as metastasis in TNBC cells [29]. Furthermore, its activation in BC induces angiogenesis in a way that is independent on VEGF activation [29]. SMO receptors and GLI1 signaling components of this pathway are highly expressed in TNBC. This overexpression is responsible of poor prognosis and recurrence of the tumor [30]. GLI1 inhibitors reduce GLI1 expression and inhibit its binding to target DNA sequences [31]. Decreased GLI1 expression has been found to reduce tumor growth and viability of breast CSCs in a xenograft model [32].

\section{Notch Signaling Pathway}

Abnormal NOTCH signaling activation initiates tumor formation and enhances angiogenesis [33]. NOTCH signaling pathway has been inhibited by GSI ( $\gamma$-secretase inhibitors) [34] NOTCH-1 has been found to be overexpressed in TNBC NOTCH-4 has a great role in initiation of TNBC [35].

\section{Targeting Receptor Tyrosine Kinases in TNBC}

RTKs are a group of cell surface receptors. They have specific structural features make them with high affinity to different polypeptides signaling molecules as: hormones, cytokines, and growth factor. It is responsible for regulation of many downstream signaling pathways as MAPK, JAK/STAT and PI3K/AKT. This regulation makes ita key factor in controlling cell growth, metabolism cell differentiation and proliferation [36]. The deregulation of these molecules has a key role in regulating tumorigenesis, angiogenesis, and metastasis. RTKs' overexpression in BC increases tumor aggressiveness and metastasis. Their multi-faceted roles make them promise molecular targets for targeted therapy in many cancers. However, gene amplification and alternate pathway activation make anti-RTK therapy challenging. The RTK family members, which are overexpressed, in TNBC are VEGFR [37], TGFßR [38], EGFR (HER1) [39], FGFR [40], PDGFR [41] and IGF-IR [42]. TKI (tyrosine kinase inhibitor), known as "Erlotinib" and acts on EGFR, increases the expression of E-Cadherin and down regulates Vimentin expression in TNBC cells. This leads to change of mesenchymal phenotype to epithelial phenotype (EMT) [43]. Other TKI agents act on EGFR as
"Cetuximab", "geftinib" and "Panitumumab" does not produce any significant results when tested clinically [44]. "Sunitinib" inhibits PDGF\& VEGF which induce reduction in tumor volume in TNBC cells when tested in xenograft models [45]. "Bevacizumab" inhibits VEGF, thus, decreases the progression of tumor in 35\% of TNBC patients [46]. "Nintedanib" is a multi-targeted angiokinase inhibitor that acts against many growth factors, as PDGFR, VEGFR and FGFR [47]. In BC, the combination of chemotherapy paclitaxel enhances tumor regression in 50\% of patients with HER2 negative BC [48]. It inhibits the effect of STAT3 activated molecules. This is the main mechanism by which Nintedanib induces apoptosis in TNBC cells. That is why it decreases the average tumor size in mice bearing MDA-MB-231 tumor.

\section{References}

1. Youness RA, MZ Gad (2019) Long non-coding RNAs: Functional regulatory players in breast cancer. Noncoding RNA Res 4(1): 36-44.

2. Bray F (2018) Global cancer statistics 2018: GLOBOCAN estimates of incidence and mortality worldwide for 36 cancers in 185 countries. CA Cancer J Clin 68(6): 394-424.

3. Youness RA (2018) A novel role of sONE/NOS3/NO signaling cascade in mediating hydrogen sulphide bilateral effects on triple negative breast cancer progression. Nitric Oxide 80: 12-23.

4. Youness RA (2019) The long noncoding RNA sONE represses triplenegative breast cancer aggressiveness through inducing the expression of miR-34a, miR-15a, miR-16, and let-7a. J Cell Physiol 234(11): 2028620297.

5. Lehmann BD (2011) Identification of human triple-negative breast cancer subtypes and preclinical models for selection of targeted therapies. J Clin Invest 121(7): 2750-2767.

6. Ahn SG (2016) Molecular Classification of Triple-Negative Breast Cancer J Breast Cancer 19(3): 223-230.

7. Prado Vazquez G (2019) A novel approach to triple-negative breast cancer molecular classification reveals a luminal immune-positive subgroup with good prognoses. Sci Rep 9(1): 1538.

8. Xiao Y (2019) Multi-Omics Profiling Reveals Distinct Microenvironment Characterization and Suggests Immune Escape Mechanisms of TripleNegative Breast Cancer. Clin Cancer Res 25(16): 5002-5014.

9. Awad AR (2019) An acetylated derivative of vitexin halts MDA-MB-231 cellular progression and improves its immunogenic profile through tuning miR- 20a-MICA/B axis. Nat Prod Res pp. 1-5.

10. Senkus E (2015) Primary breast cancer: ESMO Clinical Practice Guidelines for diagnosis, treatment, and follow-up. Ann Oncol 26(Suppl 5): v8-30.

11. von Minckwitz G (2012) Definition and impact of pathologic complete response on prognosis after neoadjuvant chemotherapy in various intrinsic breast cancer subtypes. J Clin Oncol 30(15): 1796-1804.

12. Lebert JM (2018) Advances in the systemic treatment of triple-negative breast cancer. Curr Oncol 25(Suppl 1): S142-S150.

13. Masuda N (2017) Adjuvant Capecitabine for Breast Cancer after Preoperative Chemotherapy. N Engl J Med 376(22): 2147-2159.

14. Denduluri N (2016) Selection of Optimal Adjuvant Chemotherapy Regimens for Human Epidermal Growth Factor Receptor 2 (HER2) -Negative and Adjuvant Targeted Therapy for HER2-Positive Breast Cancers: An American Society of Clinical Oncology Guideline Adaptation of the Cancer Care Ontario Clinical Practice Guideline. J Clin Oncol 34(20): 2416-2427. 
15. Chalakur Ramireddy NKR, SB Pakala (2018) Combined drug therapeutic strategies for the effective treatment of Triple Negative Breast Cancer. Biosci Rep 38(1)

16. Ossovskaya V (2011) Exploring molecular pathways of triple-negative breast cancer. Genes Cancer, 2(9): 870-879.

17. Pan H (2012) Genistein inhibits MDA-MB-231 triple-negative breast cancer cell growth by inhibiting NF-kappaB activity via the Notch-1 pathway. Int J Mol Med 30(2): 337-343.

18. Ahmad A (2008) Plumbagin-induced apoptosis of human breast cancer cells are mediated by inactivation of NF-kappaB and Bcl-2. J Cell Biochem 105(6): 1461-1471.

19. Li T (2014) Fenofibrate induces apoptosis of triple-negative breast cancer cells via activation of NF-kappaB pathway. BMC Cancer 14: 96.

20. Wei W (2014) STAT3 signaling is activated preferentially in tumorinitiating cells in claudin-low models of human breast cancer. Stem Cells 32(10): 2571-2582.

21. Balko JM (2016) Triple-negative breast cancers with amplification of JAK2 at the 9p24 locus demonstrate JAK2-specific dependence. Sci Transl Med 8(334): 334ra53.

22. Deng XS (2012) Metformin targets Stat3 to inhibit cell growth and induce apoptosis in triple-negative breast cancers. Cell Cycle 11(2): 367-376.

23. Gordon V, S Banerji (2013) Molecular pathways: PI3K pathway targets in triple-negative breast cancers. Clin Cancer Res 19(14): 3738-3744.

24. Park JH (2016) PI3K/Akt/mTOR activation by suppression of ELK3 mediates chemosensitivity of MDA-MB-231 cells to doxorubicin by inhibiting autophagy. Biochem Biophys Res Commun 477(2): 277-282.

25. Yunokawa M (2012) Efficacy of everolimus, a novel mTOR inhibitor, against basal-like triple-negative breast cancer cells. Cancer Sci 103(9): 1665-1671.

26. Khramtsov AI (2010) Wnt/beta-catenin pathway activation is enriched in basal-like breast cancers and predicts poor outcome. Am J Pathol 176(6): 2911-2920.

27. Bilir B, O Kucuk, CS Moreno (2013) Wnt signaling blockage inhibits cell proliferation and migration and induces apoptosis in triple-negative breast cancer cells. J Transl Med 11: 280

28. MacDonald BT, K Tamai, X He (2009) Wnt/beta-catenin signaling: components, mechanisms, and diseases. Dev Cell 17(1): 9-26.

29. Harris LG (2012) Increased vascularity and spontaneous metastasis of breast cancer by hedgehog signaling mediated upregulation of cyr61. Oncogene 31(28): 3370-3380.

30. Tao Y (2011) Overexpression of Hedgehog signaling molecules and its involvement in triple-negative breast cancer. Oncol Lett 2(5): 995-1001.

31. Lauth M (2007) Inhibition of GLI-mediated transcription and tumor cell growth by small-molecule antagonists. Proc Natl Acad Sci U S A 104(20): 8455-8460.

32. Colavito SA (2014) Significance of glioma-associated oncogene homolog 1 (GLI1) expression in claudin-low breast cancer and crosstalk with the nuclear factor kappa-light-chain-enhancer of activated B cells (NFkappaB) pathway. Breast Cancer Res 16(5): 444.

33. Phng LK, H Gerhardt (2009) Angiogenesis: a team effort coordinated by notch. Dev Cell 16(2): 196-208.

34. Li ZL (2015) Gamma secretase inhibitor enhances sensitivity to doxorubicin in MDA-MB-231 cells. Int J Clin Exp Pathol 8(5): 4378-4387.

35. Nagamatsu I (2014) NOTCH4 is a potential therapeutic target for triplenegative breast cancer. Anticancer Res 34(1): 69-80.

36. Zhang J, SN Hochwald (2013) Targeting receptor tyrosine kinases in solid tumors. Surg Oncol Clin N Am 22(4): 685-703.

37. Dent SF (2009) The role of VEGF in triple-negative breast cancer: where do we go from here? Ann Oncol 20(10): 1615-1617.

38. Jovanovic B (2014) Transforming growth factor beta receptor type III is a tumor promoter in mesenchymal-stem like triple negative breast cancer. Breast Cancer Res 16(4): R69.

39. Song H (2013) Targeting aberrant DNA double-strand break repair in triple-negative breast cancer with alpha-particle emitter radiolabeled anti-EGFR antibody. Mol Cancer Ther 12(10): 2043-2054.

40. Sharpe R (2011) FGFR signaling promotes the growth of triple-negative and basal-like breast cancer cell lines both in vitro and in vivo. Clin Cancer Res 17(16): 5275-5286.

41. Carvalho I (2005) Overexpression of platelet-derived growth factor receptor alpha in breast cancer is associated with tumour progression. Breast Cancer Res 7(5): R788-795.

42. Litzen Burger BC (2011) High IGF-IR activity in triple-negative breast cancer cell lines and tumor grafts correlates with sensitivity to anti-IGFIR therapy. Clin Cancer Res 17(8): 2314-2327.

43. Ueno NT, D Zhang (2011) Targeting EGFR in Triple Negative Breast Cancer. J Cancer 2: 324-328.

44.Amin DN (2010) Resiliency and vulnerability in the HER2-HER3 tumorigenic driver. Sci Transl Med 2(16): 16 ra7.

45. Chinchar E (2014) Sunitinib significantly suppresses the proliferation, migration, apoptosis resistance, tumor angiogenesis and growth of triple-negative breast cancers but increases breast cancer stem cells. Vasc Cell 6: 12.

46. O Shaughnessy J (2010) Abstract P6-12-03: Meta-Analysis of Patients with Triple-Negative Breast Cancer (TNBC) from Three Randomized Trials of First-Line Bevacizumab (BV) and Chemotherapy Treatment for Metastatic Breast Cancer (MBC). Cancer Research 70(24): P6-12.

47. Reck M (2015) Nintedanib: examining the development and mechanism of action of a novel triple angiokinase inhibitor. Expert Rev Anticancer Ther 15(5): 579-594.

48. Quintela Fandino M (2014) Phase I clinical trial of Nintedanib plus paclitaxel in early HER-2-negative breast cancer (CNIO-BR-01-2010/ GEICAM-2010-10 study). Br J Cancer 111(6): 1060-1064. 
ISSN: 2574-1241

DOI: $10.26717 /$ BJSTR.2020.29.004757

Rana Ahmed Youness. Biomed J Sci \& Tech Res

(C) (P) This work is licensed under Creative BY Commons Attribution 4.0 License

Submission Link: https://biomedres.us/submit-manuscript.php

$\begin{array}{ll}\text { BIOMEDICAL } & \text { Assets of Publishing with us } \\ \text { RESEARCHES } & \text { - Global archiving of articles } \\ \text { - Immediate, unrestricted online access } & \text { - Rigorous Peer Review Process } \\ & \text { - Authors Retain Copyrights } \\ & \end{array}$

